Research Paper

\title{
The alterations of degree centrality in the frontal lobe of patients with panic disorder
}

\author{
Yongbao Liu1, Chien-Han Lai2,3凶 \\ 1. Department of Imaging, The First People's Hospital of LianYun Gang, Lianyungang City, Jiangsu Province, 222000, China \\ 2. Institute of Biophotonics, National Yang-Ming University, Taipei, Taiwan \\ 3. PhD Psychiatry \& Neuroscience Clinic, Taoyuan, Taiwan \\ $\triangle$ Corresponding author: Chien-Han Lai, MD, PhD. Institute of Biophotonics, National Yang-Ming University, No.155, Sec.2, Linong Street, Taipei, 112 Taiwan \\ (ROC), Telephone: 886-2-2826-7000 ext 5707, Fax: 886-2-2823-5460, Email: stephenlai99@gmail.com
}

(C) The author(s). This is an open access article distributed under the terms of the Creative Commons Attribution License (https://creativecommons.org/licenses/by/4.0/). See http://ivyspring.com/terms for full terms and conditions.

Received: 2021.07.26; Accepted: 2021.11.02; Published: 2022.01.01

\begin{abstract}
Objective: The brain network in panic disorder (PD) is still an intriguing issue for research. In this study, we hoped to investigate the role of DC (degree centrality) for the pathophysiology of PD, especially for the fear network.

Methods: We enrolled 60 patients with PD and 60 controls in the current study. The gender and age were matched for two groups. All participants received the resting-state functional magnetic resonance imaging to survey the baseline brain activity. Then the DC values of all participants were using REST toolbox. We also compared the DC values between PD and controls. The statistical threshold was set as FDR (false discovery rate) $<0.05$.

Results: The DC values were significantly lower in the right superior frontal gyrus of PD patients compared to controls (FDR < 0.05). In addition, a negative correlation between the DC values and panic severity was observed in the right superior frontal gyrus and left inferior frontal gyrus. However, there was no significant association between the DC values and illness duration.

Conclusion: The DC seemed significantly altered in the frontal lobe of PD patients. The role of the frontal lobe might be more emphasized in the pathophysiology research for PD.
\end{abstract}

Key words: panic disorder; degree centrality; superior frontal gyrus; inferior frontal gyrus, alterations

\section{Introduction}

Panic disorder (PD) is characterized of autonomic nervous system dysfunction, such as dizziness, chest tightness, palpitations and other somatic complaints. The neuroanatomy hypothesis of PD basically focused on the fear network hypothesis, including the frontal lobe, thalamus, amygdala, hippocampus, hypothalamus and brainstem [1]. In recent years, another review article of PD hypothesis suggested the potential extension of fear circuit areas into more regions, such as the anterior cingulate and insula. [2].

The default brain activity has been applied in the neuroscience research for recent one decade. It can be evaluated by the resting-state functional magnetic resonance imaging (Rs-FMRI) technique. There are many parameters for the measurement of default brain activity. Among the parameters, the degree centrality (DC), a measurement of centrality, connectedness and influences of a node within a network. The node's prominence profile can reveal the network dynamics and node centrality [3]. For the viewpoint of Rs-FMRI, the DC can provide the information for the number of instantaneous functional connections between a region and the rest of the brain within the entire brain connectome. Therefore the DC values can reveal the node influences for entire brain areas [4]. It has been applied in the pathophysiology study for many kinds of illness, such as depression [5-7], schizophrenia [8], bipolar disorder [9] and end-stage renal disease [10]. The application of DC study in anxiety pathophysiology is rare [11]. For the PD, the role of 
DC has not been addressed much.

In the fear network model for PD, the crucial role of frontal cortex and limbic regions has been proved in the several studies. The blood perfusions were altered in the frontal and limbic regions, such as hippocampus, in the patients with PD [12]. The benzodiazepine receptor uptake was reduced in the frontal cortex of PD patients [13]. PD patients also showed increased brain activities in the left inferior frontal cortex and hippocampus when exposing to emotion-related memory [14]. Our previous report of remitted PD patients also demonstrated the changes in the regional homogeneity of frontal and temporal regions after antidepressant treatment [15]. These reports supported the importance of frontal cortex and limbic regions in the PD pathophysiology.

In current study, we wanted to utilize the DC to survey the altered pattern in the patients with PD. From the fear network hypothesis and the literature mentioned above, we hypothesized that the alterations of DC would be observed in the frontal cortex and limbic regions. The possible correlations between panic severity, duration of illness and DC values might be also found the frontal cortex and limbic regions.

\section{Methods}

All patients and healthy subjects signed the informed consent that was approved by the three Institutional Review Boards (Taipei Tzu Chi General Hospital, Cheng Hsin General Hospital and National Yang-Ming University) according to the institute where they were recruited. Informed consent was obtained from all of the individual participants included in the study. None of PD patients had comorbid depression. The PD group was enrolled according to the following criteria: (1) first-episode patients with a pure PD diagnosis (DSM-IV criteria) (2) The severity of PD was at least moderate: Clinician Global Impression of Severity > 4, Quick Inventory for Depressive Symptoms-Self Rating 16-item version (QIDS-SR16) < 9, Hamilton Rating Scales for Depression (HDRS) score $<7$, Hamilton Rating Scales for Anxiety (HARS) score $>22$, Panic Disorder Symptom Severity Scale (PDSS) $>15$, panic attacks of full blown symptom $>4$ times within previous 4 weeks before the baseline visit. (3) no co-morbid psychiatric illnesses or medical illnesses; (4) no previous cognitive behavioural therapy or other psychotherapies; (5) medication-naïve; (6) no abuse of or dependence on alcohol or other substances; and (7) no past history of claustrophobia or discomfort while receiving MR scanning. The healthy controls had no psychiatric illnesses or significant medical illnesses. The patients were enrolled at two general hospitals.
The controls were enrolled from 2 general hospitals and one university. At the time of the MR imaging, none of the participants in the control group received psychotropic treatment. Handedness was determined by using the Edinburgh Inventory of Handedness [16]. We screened 101 patients with PD and 67 controls. Finally, we enrolled 60 patients with PD and 60 controls according to the above selection criteria.

\section{Structural MRI (magnetic resonance imaging) data acquisition}

The structural MRI T1 imaging scans of brain were obtained with 3T Siemens version scanner housed at MRI Center, National Yang Ming University. Scans with three-dimensional fast spoiled gradient-echo recovery (3D-FSPGR) T1W1 (TR $25.30 \mathrm{~ms}$; TE $3.03 \mathrm{~ms}$; slice thickness $=1 \mathrm{~mm}$ (no gap); 192 slices; matrix $=224 \times 256$; field of view: $256 \mathrm{~mm}$; number of excitation=1) were performed.

\section{Rs-FMRI data acquisition and pulse sequence}

Echo planar imaging (EPI) sequence were acquired in 20 axial slices $(T R=2000 \mathrm{~ms}$, TE $=40 \mathrm{~ms}$, flip angle $=90^{\circ}$, field of view $=24 \mathrm{~cm} ; 5 \mathrm{~mm}$ thickness and 1 $\mathrm{mm}$ gap; the sequence duration was 300 seconds for each subject, 150 time points were acquired, voxel dimension: $64 \times 64 \times 20)$ at baseline visit (3T Siemens scanner housed at MR center of National Yang Ming University) in patients and controls. All the patients and controls were requested to close their eyes with relaxing manner and not sleepy while scanning. The participating subjects were instructed to move as little as possible and stay fully awake while scanning. All these patients and controls reported that they could be fully awake while MRI scanning.

\section{Rs-FMRI preprocessing and DC analysis}

EPI data was first preprocessed by DPARSF (Data Processing Assistant and Resting-State FMRI, version 2.2; State Key Laboratory of Cognitive Neuroscience and Learning, Beijing Normal University, Beijing, China.) [17] working with the statistical parametric mapping 8 (SPM8) on the Matlab platform, which included the removal of first 10 time points due to the consideration for instability of initial MRI signal and patients' difficult to adapt at first about MRI acquisition circumstance, slice timing with $20^{\text {th }}$ slice as reference slice, realignment, normalization to standard MNI spaces by using EPI templates and re-sampling with $3 \times 3 \times 3 \mathrm{~mm} 3$, smoothing by Full Width at Half Maximum (FWHM) $4 \times 4 \times 4$ kernel, to detrend and filter data with residual signals within $0.01-0.08 \mathrm{~Hz}$ to discard the bias from high-frequency physiological noise and lowfrequency drift. As all subjects' head movements were less than $0.5 \mathrm{~mm}$ in translation and 1 degree in 
rotation by obtaining the motion time courses of all subjects, no subject was excluded due to no excessive motions were observed. The realignment estimates were also used for reducing motion contamination for the data [18]. A study-specific MNI template was derived from segmented transformed images consisting of gray matter, white matter and cerebrospinal fluid into the MNI space. The filtered RFMRI data was registered (nonlinear elastric registration) to this symmetric study-specific MNI template, which was created by voxel-based morphometry of T1 images. The estimated motion parameters were obtained for each subject regressed on each voxel. Non-gray matter nuisances, such as the white matter and CSF signals, were regressed to reduce the motion artifacts [18]. Several sources of spurious covariates were removed except global signals due to the controversy about removing the global signal in the resting-state functional connectivity data $[19,20]$. The global signal regression method combined with within-subject and censoring-based artifact removal strategy [21] were also used for reducing motion artifacts. The individual-level covariates of motion included Friston-24 parameter model $(6$ head motion parameters, 6 head motion parameters one time point before, and the 12 corresponding squared items) and group-level covariates of motion included framewise displacement motion regression model [22]. We also implemented some denoising procedure that produces incompletely denoised time series (e.g., mean white matter signal regression), and then to use partial correlations rather than full correlations to study relationships among the time series. The final output data after DPARSF preprocessing was then processed by REST (Resting State FMRI Data Analysis Toolkit, version 1.4; State Key Laboratory of Cognitive Neuroscience and Learning, Beijing Normal University, Beijing, China.) [23] and DC data was produced subsequently.

The DC values of all subjects were produced by the REST toolbox. The whole-brain functional connectivity matrix would be produced using Pearson's correlation coefficients between the time series of all pairs of gray matter voxels. This computation followed the gray matter mask set by the previous article [24]. The Fisher's r-to-z transformation was used to derive the Z-score matrix and improve normality. The regional functional connectivity strength was calculated as the sum of all the connections (Z-values) between voxels. The analysis was conservatively restricted to positive correlations above a threshold of $r=0.2$, which can eliminate the weak correlation due to signal noise. We identified the voxels with higher regional functional connectivity strength values, which usually indicate the centrality within the specific network of brain.

\section{Statistical analysis}

The DC map was used for the following independent two sample $t$ test analysis function implemented in the REST toolbox. The subsequent group comparison analysis between patients and controls was performed (second-level random effects model, independent two sample $t$ test) under the following statistical criteria [false discovery rate (FDR) corrected $\mathrm{p}<0.005$, cluster $>10$ voxels, $\mathrm{T}$ threshold: 5.1847, surface connected theory]. The above statistical analysis also used age and gender as covariates to exclude possible influences from these parameters. In addition, to clarify the relationship between clinical severity of panic symptoms and DC values, a voxel-wise Pearson's correlation was performed. For each subject, the Pearson correlation coefficient between each voxel's residual time series and that of its symmetrical inter-hemispheric counterpart was performed. The correlation values were then Fisher z-transformed to improve normality.

\section{Results}

\section{Demographic data}

There were no significant differences in age, gender, HDRS scores between the patient and control groups. However, significant differences were observed in the HARS scores and PDSS scores between patients and controls. All participants of the two groups were almost right-handed (SPSS version 16).

\section{The differences in the $\mathrm{DC}$ values between patients and controls}

The PD patients had significantly lower DC values in the right SFG (FDR corrected $\mathrm{p}<0.05$ ) (Table 2, upper panel of Figure 1). In addition, negative correlations between the DC values and panic severity were observed in the right SFG and left inferior frontal gyrus (IFG) (Table 2, lower panel of Figure 1). However, there was no significant association between the DC values and illness duration.

Table 1: Demographic data of participating patients and controls

\begin{tabular}{|c|c|c|c|}
\hline & $\begin{array}{l}\text { Patients } \\
(\mathrm{N}=60)\end{array}$ & $\begin{array}{l}\text { Controls } \\
(\mathrm{N}=60)\end{array}$ & $\begin{array}{l}\text { Sig } p \\
\text { (2-tailed), } \mathrm{Z} \\
\mathrm{df}=118\end{array}$ \\
\hline Age, mean (SD), years old & $43.28(9.66)$ & $\begin{array}{l}40.71 \\
(10.38)\end{array}$ & $0.109,-1.61$ \\
\hline Gender (number) & $\begin{array}{l}\mathrm{F}(30), \\
\mathrm{M}(30)\end{array}$ & $\begin{array}{l}\mathrm{F}(30), \\
\mathrm{M}(30)\end{array}$ & 0.715 \\
\hline Duration of illness, mean (SD), months & $5.46(2.28)$ & $0(0)$ & $\mathrm{N} / \mathrm{A}$ \\
\hline Educational years, mean (SD) & $15.96(1.05)$ & $\begin{array}{l}16.23 \\
(0.83)\end{array}$ & $0.102,-1.636$ \\
\hline Handedness & R (58), L (2) & \multicolumn{2}{|c|}{$\mathrm{R}(58), \mathrm{L}(2) 0.152$} \\
\hline
\end{tabular}




\begin{tabular}{llcl}
\hline & $\begin{array}{l}\text { Patients } \\
(\mathrm{N}=60)\end{array}$ & $\begin{array}{l}\text { Controls } \\
(\mathrm{N}=60)\end{array}$ & $\begin{array}{l}\text { Sig p } \\
(2 \text {-tailed), } \mathrm{Z} \\
\mathrm{df}=118\end{array}$ \\
\hline HDRS, mean (SD) & $1.15(0.86)$ & $0.96(0.89)$ & $0.21,-1.603$ \\
HARS, mean (SD) & $23.21(2.66)$ & $\begin{array}{l}1.22 \\
(0.91)\end{array}$ & $<0.001,-9.571$ \\
PDSS, mean (SD) & $21.40(1.83)$ & $\mathrm{N} / \mathrm{A}$ & $\mathrm{N} / \mathrm{A}$ \\
\hline
\end{tabular}

$\mathrm{N}$ : number; SD: standard deviation; F: female, M: male; HDRS: Hamilton rating scales for depression; HARS: Hamilton rating scales for anxiety; PDSS: panic disorder severity scale; N/A: not applicable; Sig p (significance of p-value) was from Mann-Whitney $U$ test for nonparametric independent 2-sample t-test; df: degree of freedom.

Table 2. The DC alterations and negative correlations between $\mathrm{DC}$ and panic severity in the frontal lobe of PD patients

\begin{tabular}{llll}
\hline Region & $\begin{array}{l}\text { MNI } \\
\text { coordinate } \\
\mathrm{x} \mathrm{y} \mathrm{z}\end{array}$ & $\begin{array}{l}\text { Cluster } \\
\text { voxels }\end{array}$ & Statistical significance \\
\hline Right SFG & $27,66,6$ & 47 & $\begin{array}{l}\text { FDR corrected } \mathrm{p}<0.05 \text {, peak intensity: } \\
5.0685 \\
\text { FDR corrected } \mathrm{p}<0.05, \mathrm{r}:-0.51\end{array}$ \\
$\begin{array}{l}\text { Right SFG } \\
\text { (correlation) }\end{array}$ & $27,65,4$ & 50 & FDR corrected $\mathrm{p}<0.05, \mathrm{r}:-0.42$ \\
$\begin{array}{l}\text { Left IFG } \\
\text { (correlation) }\end{array}$ & $-53,37,4$ & 41 & \\
\hline
\end{tabular}

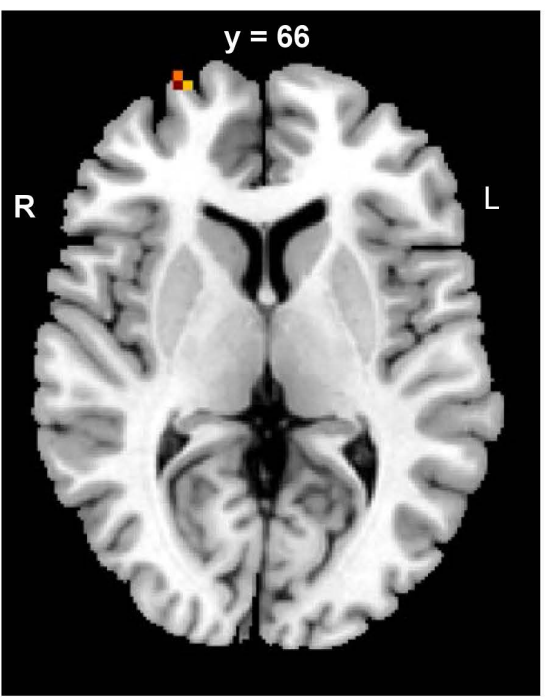

5.09

$-4.05$

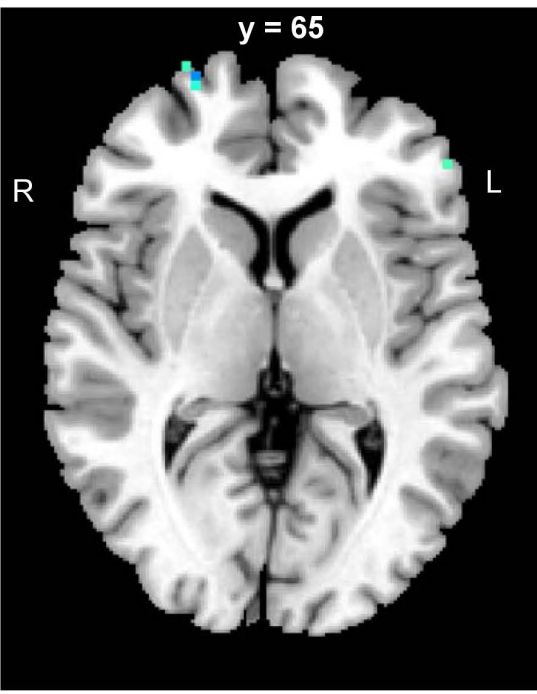

$-5.18$

0.58

0.32

$-0.51$

Figure 1: The alterations of frontal cortex in DC values of PD patients. The PD patients had lower values of DC in SFG when compared to controls (upper panel figure). In addition, the negative correlations between panic severity and $D C$ values were observed in the right SFG and left IFG (lower panel figure). (MNI: Montreal Neurological Institute; SFG: superior frontal gyrus; IFG: inferior frontal gyrus)

\section{Discussion}

We found significant alterations in the DC values of frontal cortex, such as the right SFG in current study. However, the absence of DC alterations in the limbic system was incompatible with our hypothesis. In addition, we found significantly negative correlation between DC values and panic severity in the right SFG and left IFG. Such a correlation was not found between illness duration and DC values.

The alteration of DC in the frontal lobe, such as the right SFG, was the major finding in current study. In the panic-provoking model, it indicated that the activation of right SFG might play a role in the dysregulation of fear network model in PD [25]. Another finding is the negative correlation of panic severity with the DC values in the left IFG and right SFG. This finding is also compatible with the antidepressant-related modulations in the metabolic and regional homogeneity in the frontal regions of PD patients [15, 26]. In addition, the stimulation of SFG also can modulate inhibitory control for motor response, which might be important for the panic responses using the motor muscles [27]. Our study result of left SFG also corresponded to the inhibitory control hypothesis of PD [27]. The SFG can regulate goal-directed top-down process and is a part of dorsal attention system [28]. According to the study of resting-state functional connectivity, the SFG is connected with the thalamus and other limbic regions, which are also components of fear circuitry of PD [29]. However, this theory might need further resting-state function connectivity to support our results of DC alterations in SFG. In the previous model of short-term treatment of paroxetine for PD, the post-treated patients showed significant changes of glucose metabolisms in frontal regions [26]. It supported the crucial role of frontal region in the pathophysiology and clinical responses in PD. From the above literature, SFG might interact with other limbic regions within fear circuitry for inhibition of panic responses, which also suggest the important role of SFG in etiology of PD. However, current study results found no significant alterations of DC values in the limbic system. It can be explained as that maybe DC values might not be such sensitive as a biomarker for the alterations in limbic system of PD patients, even without abundant study findings of DC in PD can support this explanation. Maybe researchers can perform more DC studies in PD and focus on the limbic system in the future. If we combine the above literature with the findings in current study, it suggests that the SFG might play the role for the trait-dependent biomarker for DC, which may constitute the blueprint of network centrality and stability of PD. 
The negative relationship between DC values and panic severity can be an interesting point for understanding the role of DC in the pathophysiology of PD. From the structural point, it indicated the importance of IFG in the pathophysiology of PD and the relation to the severity of PD [30-32]. The panic-specific pictures will also induce greater activation in the IFG and limbic regions in PD patients [33]. The later study also replicated the findings of IFG in the responses to the panic-related pictures in PD patients [34]. The IFG-related inhibitory control and impacts to the fear network circuitry might be also associated with the panic response [35]. The right-left asymmetry and decreased activities of cerebral blood flow in the IFG was also found during panic attacks $[12,36]$, which might also explain the correlation of DC values with the panic severity. The impairments of cognitive function [37] and hypofrontality was also found in the left IFG of PD patients [38]. The alteration of functional MRI signals in the IFG was also observed during the processing of panic-related stimulus [39] and the recognition of negative words [40]. The functional MRI study of cognitive behavioral therapy also showed that the IFG might be cognitive processing core for the improvement in panic syndrome. In contrast, the typical fear network was responsible for emotional processing in the remission of panic symptoms [41]. Therefore from our study results, the correlation between DC values and panic severity may suggest that the role of DC values in the pathophysiology of PD.

Our study is the first study of DC values in PD patients. We also found the important role of frontal regions, such as SFG and IFG, in the DC pathophysiology model for PD. Our study results can advance our understandings about the formation of PD from the viewpoint of DC, especially for the frontal region within the fear network model. The SFG and IFG are the crucial regions for cognitive function. The study of antidepressant treatment showed the treatment response accompanied with the changes in glucose metabolism in the IFG and SFG[42], which might play a role in the explanation of current study results. Another study also demonstrated the involvement of IFG in the response of antidepressant therapy in PD [26]. PD patients might also have a prolonged activation in the left IFG during extinction recall [43]. The negative correlation of DC values with panic severity in the IFG might be associated with this kind of involvement. The functional MRI study of panic-related words and emotional Stroop task showed that the processing function of the attention and emotion would be associated with alterations of activities in the IFG of PD patients [39]. The emotional word recognition task also provoked the heightened signals in the IFG [40]. The functional MRI study of cognitive behavioral therapy in PD suggested that the cerebral correlates of IFG would interact with the limbic regions in the improvements of PD symptoms [41], which corresponded to the decoupling sign between IFG and limbic regions of another study [44]. The transcranial magnetic stimulation study also replicated the importance of IFG in the treatment response in PD [45]. The anxiety-related trait and dimension also point to the IFG [46]. From the above literature, the SFG and IFG may be the core region for the top-down mechanism of pathophysiology in PD [47]. Our findings in current study might support the crucial role of SFG and IFG in the pathophysiology of PD. The relationship with the panic severity might suggest the potential biomarker for PD. However, the further study with a well-designed architecture can help us confirm the hypothesis.

First, the cross-sectional design is a major weakness. A future longitudinal study would help use confirm the importance of DC in the IFG and SFG of PD patients. Second, the lack of cognitive function and neuropsychological data in current sample might limit explanation of cognitive core of IFG and SFG and the relationship with DC values for PD. Third, functional connectome measures the functional connectivity according to the signals of hemodynamics. The alterations of DC values are probably related to the hemodynamic changes. However, it is still unknown whether the method of DC can be used to detect early neuronal changes, or monitor disease progression in PD. Fourth, a task-oriented functional MRI study could complete the viewpoint due to Rs-FMRI characteristics of the functional connectome results. A future study combined with task-oriented functional MRI method can help us confirm the findings of DC values in current study.

\section{Conclusion}

From current study results, the crucial role of frontal lobe in PD can be proved in the study of DC alterations. The SFG might be an important biomarker for DC. In addition, the negative correlation of DC values with panic severity in the IFG might be another crucial part for the DC alterations in PD patients.

\section{Competing Interests}

The authors have declared that no competing interest exists.

\section{Acknowledgements}

We want to thank the grant support from Taipei Tzu-Chi General Hospital project TCRD-TPE-99-02 
and the project was also supported by a grant from the Cheng Hsin General Hospital and National Yang Ming University cooperative project 104F003C03.

\section{References}

1. Gorman JM, Kent JM, Sullivan GM, Coplan JD. Neuroanatomical hypothesis of panic disorder, revised. Am J Psychiatry. 2000; 157: 493-505.

2. Dresler T, Guhn A, Tupak SV, Ehlis AC, Herrmann MJ, Fallgatter AJ, et al. Revise the revised? New dimensions of the neuroanatomical hypothesis of panic disorder. J Neural Transm (Vienna). 2013; 120: 3-29.

3. Yang Y, Dong Y, Chawla NV. Predicting node degree centrality with the node prominence profile. Scientific reports. 2014; 4: 7236.

4. Takeuchi H, Taki Y, Nouchi R, Sekiguchi A, Hashizume H, Sassa Y, et al. Degree centrality and fractional amplitude of low-frequency oscillations associated with Stroop interference. NeuroImage. 2015; 119: 197-209.

5. Gao C, Wenhua L, Liu Y, Ruan X, Chen X, Liu L, et al. Decreased Subcortical and Increased Cortical Degree Centrality in a Nonclinical College Student Sample with Subclinical Depressive Symptoms: A Resting-State fMRI Study. Frontiers in human neuroscience. 2016; 10: 617.

6. Guo Z, Liu X, Hou H, Wei F, Liu J, Chen X. Abnormal degree centrality in Alzheimer's disease patients with depression: A resting-state functional magnetic resonance imaging study. Exp Gerontol. 2016; 79: 61-6.

7. Shen Y, Yao J, Jiang X, Zhang L, Xu L, Feng R, et al. Sub-hubs of baseline functional brain networks are related to early improvement following two-week pharmacological therapy for major depressive disorder. Hum Brain Mapp. 2015; 36: 2915-27.

8. Chen C, Wang HL, Wu SH, Huang H, Zou JL, Chen J, et al. Abnormal Degree Centrality of Bilateral Putamen and Left Superior Frontal Gyrus in Schizophrenia with Auditory Hallucinations: A Resting-state Functional Magnetic Resonance Imaging Study. Chinese medical journal. 2015; 128: 3178-84.

9. Zhou Q, Womer FY, Kong L, Wu F, Jiang X, Zhou Y, et al. Trait-Related Cortical-Subcortical Dissociation in Bipolar Disorder: Analysis of Network Degree Centrality. The Journal of clinical psychiatry. 2016

10. Li S, Ma X, Huang R, Li M, Tian J, Wen H, et al. Abnormal degree centrality in neurologically asymptomatic patients with end-stage renal disease: A resting-state fMRI study. Clinical neurophysiology : official journal of the International Federation of Clinical Neurophysiology. 2016; 127: 602-9.

11. Kajimura S, Kochiyama T, Nakai R, Abe N, Nomura M. Fear of negative evaluation is associated with altered brain function in nonclinical subjects. Psychiatry Res. 2015; 234: 362-8.

12. De Cristofaro MT, Sessarego A, Pupi A, Biondi F, Faravelli C. Brain perfusion abnormalities in drug-naive, lactate-sensitive panic patients: a SPECT study. Biological psychiatry. 1993; 33: 505-12.

13. Kuikka JT, Pitkanen A, Lepola U, Partanen K, Vainio P, Bergstrom KA, et al. Abnormal regional benzodiazepine receptor uptake in the prefrontal cortex in patients with panic disorder. Nuclear medicine communications. 1995; 16: 273-80

14. Bystritsky A, Pontillo D, Powers M, Sabb FW, Craske MG, Bookheimer SY. Functional MRI changes during panic anticipation and imagery exposure. Neuroreport. 2001; 12: 3953-7.

15. Lai $\mathrm{CH}, \mathrm{Wu} \mathrm{YT}$. Frontal regional homogeneity increased and temporal regional homogeneity decreased after remission of first-episode drug-naive major depressive disorder with panic disorder patients under duloxetine therapy for 6 weeks. Journal of affective disorders. 2012; 136: 453-8.

16. Oldfield RC. The assessment and analysis of handedness: the Edinburgh inventory. Neuropsychologia. 1971; 9: 97-113.

17. Chao-Gan Y, Yu-Feng Z. DPARSF: A MATLAB Toolbox for "Pipeline" Data Analysis of Resting-State fMRI. Frontiers in systems neuroscience. 2010; 4: 13.

18. Power JD, Schlaggar BL, Petersen SE. Recent progress and outstanding issues in motion correction in resting state fMRI. NeuroImage. 2015; 105: 536-51.

19. Fox MD, Snyder AZ, Vincent JL, Corbetta M, Van Essen DC, Raichle ME. The human brain is intrinsically organized into dynamic, anticorrelated functional networks. Proceedings of the National Academy of Sciences of the United States of America. 2005; 102: 9673-8.

20. Murphy K, Birn RM, Handwerker DA, Jones TB, Bandettini PA. The impact of global signal regression on resting state correlations: are anti-correlated networks introduced? NeuroImage. 2009; 44: 893-905.
21. Power JD, Mitra A, Laumann TO, Snyder AZ, Schlaggar BL, Petersen SE. Methods to detect, characterize, and remove motion artifact in resting state fMRI. NeuroImage. 2014; 84: 320-41.

22. Yan CG, Craddock RC, He Y, Milham MP. Addressing head motion dependencies for small-world topologies in functional connectomics. Frontiers in human neuroscience. 2013; 7: 910.

23. Song XW, Dong ZY, Long XY, Li SF, Zuo XN, Zhu CZ, et al. REST: a toolkit for resting-state functional magnetic resonance imaging data processing. PloS one. 2011; 6: e25031.

24. Wang X, Xia M, Lai Y, Dai Z, Cao Q, Cheng Z, et al. Disrupted resting-state functional connectivity in minimally treated chronic schizophrenia. Schizophrenia research. 2014; 156: 150-6.

25. Eser D, Leicht G, Lutz J, Wenninger S, Kirsch V, Schule C, et al. Functional neuroanatomy of CCK-4-induced panic attacks in healthy volunteers. Human brain mapping. 2009; 30: 511-22.

26. Sim HB, Kang EH, Yu BH. Changes in Cerebral Cortex and Limbic Brain Functions after Short-Term Paroxetine Treatment in Panic Disorder: An [F]FDG-PET Pilot Study. Psychiatry Investig. 2010; 7: 215-9.

27. Hsu TY, Tseng LY, Yu JX, Kuo WJ, Hung DL, Tzeng OJ, et al. Modulating inhibitory control with direct current stimulation of the superior medial frontal cortex. Neuroimage. 2011; 56: 2249-57.

28. Liao W, Chen H, Feng Y, Mantini D, Gentili C, Pan Z, et al. Selective aberrant functional connectivity of resting state networks in social anxiety disorder. Neuroimage. 2010; 52: 1549-58.

29. Zhang S, Ide JS, Li CS. Resting-State Functional Connectivity of the Medial Superior Frontal Cortex. Cereb Cortex. 2011.

30. Yoo HK, Kim MJ, Kim SJ, Sung YH, Sim ME, Lee YS, et al. Putaminal gray matter volume decrease in panic disorder: an optimized voxel-based morphometry study. Eur J Neurosci. 2005; 22: 2089-94.

31. van Tol MJ, van der Wee NJ, van den Heuvel OA, Nielen MM, Demenescu LR, Aleman A, et al. Regional brain volume in depression and anxiety disorders. Archives of general psychiatry. 2010; 67: 1002-11.

32. Lai $\mathrm{CH}, \mathrm{Wu} \mathrm{YT}$. Fronto-temporo-insula gray matter alterations of first-episode, drug-naive and very late-onset panic disorder patients. Journal of affective disorders. 2012; 140: 285-91.

33. Engel KR, Obst $K$, Bandelow B, Dechent $\mathrm{P}$, Gruber O, Zerr I, et al. Functional MRI activation in response to panic-specific, non-panic aversive, and neutral pictures in patients with panic disorder and healthy controls. European archives of psychiatry and clinical neuroscience. 2016; 266: 557-66.

34. Lopes FL, Faria CGF, Dias GP, Mallmann MB, Mendes V, Horato N, et al. Neural correlates of negative and disease-specific emotional stimuli in panic disorder: a functional magnetic resonance imaging study. Braz J Psychiatry. 2021.

35. Duann JR, Ide JS, Luo X, Li CS. Functional connectivity delineates distinct roles of the inferior frontal cortex and presupplementary motor area in stop signal inhibition. J Neurosci. 2009; 29: 10171-9.

36. Boshuisen ML, Ter Horst GJ, Paans AM, Reinders AA, den Boer JA. rCBF differences between panic disorder patients and control subjects during anticipatory anxiety and rest. Biol Psychiatry. 2002; 52: 126-35.

37. Nishimura $Y$, Tanii H, Fukuda M, Kajiki N, Inoue $K$, Kaiya $H$, et al. Frontal dysfunction during a cognitive task in drug-naive patients with panic disorder as investigated by multi-channel near-infrared spectroscopy imaging. Neurosci Res. 2007; 59: 107-12.

38. Ohta H, Yamagata B, Tomioka H, Takahashi T, Yano M, Nakagome K, et al. Hypofrontality in panic disorder and major depressive disorder assessed by multi-channel near-infrared spectroscopy. Depress Anxiety. 2008; 25: 1053-9.

39. Dresler T, Hindi Attar C, Spitzer C, Lowe B, Deckert J, Buchel C, et al. Neural correlates of the emotional Stroop task in panic disorder patients: an event-related fMRI study. Journal of psychiatric research. 2012; 46: 1627-34.

40. van Tol MJ, Demenescu LR, van der Wee NJ, Kortekaas R, Marjan MAN, Boer JA, et al. Functional magnetic resonance imaging correlates of emotional word encoding and recognition in depression and anxiety disorders. Biological psychiatry. 2012; 71: 593-602

41. Kircher T, Arolt V, Jansen A, Pyka M, Reinhardt I, Kellermann T, et al. Effect of cognitive-behavioral therapy on neural correlates of fear conditioning in panic disorder. Biological psychiatry. 2013; 73: 93-101.

42. Prasko J, Horacek J, Zalesky R, Kopecek M, Novak T, Paskova B, et al. The change of regional brain metabolism (18FDG PET) in panic disorder during the treatment with cognitive behavioral therapy or antidepressants. Neuro endocrinology letters. 2004; 25: 340-8.

43. Schwarzmeier H, Kleint NI, Wittchen HU, Strohle A, Hamm AO, Lueken $\mathrm{U}$. Characterizing the nature of emotional-associative learning deficits in panic disorder: An fMRI study on fear conditioning, extinction training and recall. Eur Neuropsychopharmacol. 2019; 29: 306-18.

44. Straube B, Lueken U, Jansen A, Konrad C, Gloster AT, Gerlach AL, et al. Neural correlates of procedural variants in cognitive-behavioral therapy: 
a randomized, controlled multicenter FMRI study. Psychotherapy and psychosomatics. 2014; 83: 222-33.

45. Deppermann S, Vennewald N, Diemer J, Sickinger S, Haeussinger FB, Notzon S, et al. Does rTMS alter neurocognitive functioning in patients with panic disorder/agoraphobia? An fNIRS-based investigation of prefrontal activation during a cognitive task and its modulation via sham-controlled rTMS. BioMed research international. 2014; 2014: 542526

46. Shang J, Fu Y, Ren Z, Zhang T, Du M, Gong Q, et al. The common traits of the ACC and PFC in anxiety disorders in the DSM-5: meta-analysis of voxel-based morphometry studies. PloS one. 2014; 9: e93432.

47. Lueken U, Straube B, Reinhardt I, Maslowski NI, Wittchen HU, Strohle A, et al. Altered top-down and bottom-up processing of fear conditioning in panic disorder with agoraphobia. Psychological medicine. 2014; 44: 381-94. 